Stud. Univ. Babeş-Bolyai Math. 62(2017), No. 1, 3-13

DOI: $10.24193 /$ subbmath.2017.0001

\title{
Coplexes in abelian categories
}

\author{
Flaviu Pop
}

\begin{abstract}
Starting with a pair $\mathrm{F}: \mathcal{A} \rightleftarrows \mathcal{B}: \mathrm{G}$ of additive contravariant functors which are adjoint on the right, between abelian categories, and with a class $\mathcal{U}$, we define the notion of $(\mathrm{F}, \mathcal{U})$-coplex. Considering a reflexive object $U$ of $\mathcal{A}$ with $\mathrm{F}(U)=V$ projective object in $\mathcal{B}$, we construct a natural duality between the category of all $(\mathrm{F}, \operatorname{add}(U))$-coplexes in $\mathcal{A}$ and the subcategory of $\mathcal{B}$ consisting in all objects in $\mathcal{B}$ which admit a projective resolution with all terms in the class $\operatorname{add}(V)$.
\end{abstract}

Mathematics Subject Classification (2010): 16E30, 16D90.

Keywords: Adjoint functors, duality, projective resolution, coplex.

\section{Introduction}

The study of dualities between subcategories of the module categories, induced by Hom contravariant functors associated to a given bimodule, is very important in the Module Theory in order to compare some special classes of modules. Also, is very useful to generalize such dualities, between module categories, to dualities induced by a pair of adjoint functors between abelian (or, Grothendieck) categories, because they could be applied to different pairs of adjoint functors. In [7], Castaño-Iglesias generalized the notion of costar module, introduced by Colby and Fuller in [8], to the notion of costar object in Grothedieck categories. In [5], the authors extends the notion of $f$-cotilting module (see, for example, $[16]$ ) to the notion of $f$-cotilting pair of contravariant functors. In [14], it is constructed a natural duality, induced by a pair of adjoint contravariant functors between abelian categories and, applying this result to some special classes of objects, the author generalizes some of the results related to the notion of finitistic $n$-self cotilting module, introduced by Breaz in [4]. A particular case of finitistic $n$-self cotilting module is also generalized in [6]. Starting with a pair of adjoint covariant functors $\mathrm{F}: \mathcal{A} \rightleftarrows \mathcal{B}: \mathrm{G}$, between abelian categories, in [15] it is studied, inspired by some of the results obtained by Fuller in [12] on module categories, some closure properties of some full subcategories $\mathcal{C}$ and $\mathcal{D}$ such that the restrictions $\mathrm{F}: \mathcal{C} \rightleftarrows \mathcal{D}: \mathrm{G}$ induce an equivalence. In [1] and [2], it is generalized 
the concepts of $r$-costar module and Co- $\star^{n}$-module to the concepts of $r$-costar pair and $\mathrm{Co}-\star^{n}$-tuple of contravariant functors between abelian categories. Moreover, in [3], the author generalizes $\star^{s}$-modules and $\star^{n}$-modules to $\star^{s}$-tuples and $\star^{n}$-tuples of covariant functors between abelian categories.

In this paper, we extend the notion of $G$-coplex, introduced by Faticoni in [10] (see also [11, Chapter 9]) in module categories, to the notion of $(\mathrm{F}, \mathcal{U})$-coplex in arbitrary abelian categories. More exactly, starting with a pair $\mathrm{F}: \mathcal{A} \rightleftarrows \mathcal{B}: \mathrm{G}$ of additive contravariant functors, between two arbitrary abelian categories, which are adjoint on the right and with a class $\mathcal{U}$ of objects in $\mathcal{A}$, we define the notion of $(\mathrm{F}, \mathcal{U})$ coplex, associated to this pair of functors and to the considered class. Then, setting the class $\mathcal{U}$ to be the class add $(U)$, i.e. the class of all direct summands of finite direct sums of copies of $U$, for some reflexive object $U$ of $\mathcal{A}$ with $\mathrm{F}(U)=V$ being projective object in $\mathcal{B}$, we construct a natural duality between the category of all $(\mathrm{F}$, add $(U))$ coplexes in $\mathcal{A}$ and the subcategory of $\mathcal{B}$ consisting in all objects in $\mathcal{B}$ which admit a projective resolution with all terms in the class $\operatorname{add}(V)$.

\section{Preliminaries}

Throughout this paper, we consider a pair $\mathrm{F}: \mathcal{A} \rightleftarrows \mathcal{B}: \mathrm{G}$ of additive contravariant functors, between two abelian categories, which are adjoint on the right with the natural transformations of right adjunction $\delta: 1_{\mathcal{A}} \rightarrow \mathrm{GF}$ and $\zeta: 1_{\mathcal{B}} \rightarrow$ FG. We note that the natural transformations of right adjunction, $\delta$ and $\zeta$, satisfy the identities $\mathrm{F}\left(\delta_{X}\right) \circ \zeta_{\mathrm{F}(X)}=1_{\mathrm{F}(X)}$ and $\mathrm{G}\left(\zeta_{Y}\right) \circ \delta_{\mathrm{G}(Y)}=1_{\mathrm{G}(Y)}$ for all $X \in \mathcal{A}$ and for all $Y \in \mathcal{B}$. Moreover, we mention that the functors $F$ and $G$ are left exact.

The classical example of such a pair of functors is the following (see, for example, [9, Chapter 4]).

Example 2.1. Let $R$ and $S$ be two unital associative rings and let $U$ be an $(S, R)$ bimodule. If we denote by Mod- $R$ (respectively, by $S$-Mod) the category of all right $R$ (respectively, left $S$-) modules, then the pair of Hom contravariant functors induced by $U$,

$$
\Delta=\operatorname{Hom}_{R}(-, U): \operatorname{Mod}-R \rightleftarrows S \text {-Mod : } \operatorname{Hom}_{S}(-, U)=\Delta^{\prime},
$$

is a pair of right adjoint contravariant functors via the adjunction

$$
\mu_{X Y}: \operatorname{Hom}_{R}\left(X, \operatorname{Hom}_{S}(Y, U)\right) \rightarrow \operatorname{Hom}_{S}\left(Y, \operatorname{Hom}_{R}(X, U)\right)
$$

with

$$
\mu_{X Y}(f)(y): x \mapsto f(x)(y)
$$

where $X \in \operatorname{Mod}-R, Y \in S$-Mod, $x \in X, y \in Y, f \in \operatorname{Hom}_{R}\left(X, \operatorname{Hom}_{S}(Y, U)\right)$. Associated to this adjunction, the natural transformations $\delta$ and $\zeta$ are in fact the evaluation maps

$$
\delta_{X}: X \rightarrow \operatorname{Hom}_{S}\left(\operatorname{Hom}_{R}(X, U), U\right) ; \delta_{X}(x): f \mapsto f(x)
$$

and

$$
\zeta_{Y}: Y \rightarrow \operatorname{Hom}_{R}\left(\operatorname{Hom}_{S}(Y, U), U\right) ; \zeta_{Y}(y): g \mapsto g(y),
$$

where $X \in \operatorname{Mod}-R, Y \in S$-Mod, $x \in X, y \in Y, f \in \operatorname{Hom}_{R}(X, U), g \in \operatorname{Hom}_{S}(Y, U)$. 
Castaño-Iglesias, in [7], gives an example of a pair of right adjoint contravariant functors between the categories of all $G$-graded unital right $R$-modules and of all $G$-graded unital left $S$-modules, where $G$ is a group and $R$ and $S$ are two $G$-graded unital rings (see also [13]). Other examples of such pairs of functors could be found in $[14]$.

An object $X$ in $\mathcal{A}$ (respectively, in $\mathcal{B}$ ) is called $\delta$-faithful (respectively, $\zeta$-faithful)

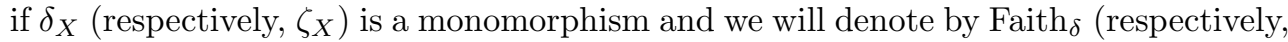
by $\mathrm{Faith}_{\zeta}$ ) the class of all $\delta$-faithful (respectively, $\zeta$-faithful) objects. An object $X$ in $\mathcal{A}$ (respectively, in $\mathcal{B}$ ) is called $\delta$-reflexive (respectively, $\zeta$-reflexive) if $\delta_{X}$ (respectively, $\zeta_{X}$ ) is an isomorphism and we will denote by $\operatorname{Refl}_{\delta}$ (respectively, by $\operatorname{Refl}_{\zeta}$ ) the class of all $\delta$-reflexive (respectively, $\zeta$-reflexive) objects.

We have the following basic results related to the closure properties of the classes of all faithful objects (see [5] for the proof).

Lemma 2.2. The following statements hold:

(a) $\mathrm{F}(\mathcal{A}) \subseteq$ Faith $_{\zeta}$ and $\mathrm{G}(\mathcal{B}) \subseteq$ Faith $_{\delta}$;

(b) The classes Faith $_{\delta}$ and Faith $_{\zeta}$ are closed with respect to subobjects.

Recall that, for a given object $X, \operatorname{add}(X)$ denotes the class of all direct summands of finite direct sums of copies of $X$. The following basic results are often used in this paper.

Lemma 2.3. Let $U$ be a $\delta$-reflexive object with $\mathrm{F}(U)=V$. Then:

(a) $V$ is $\zeta$-reflexive;

(b) $\operatorname{add}(U) \subseteq \operatorname{Refl}_{\delta}$ and $\operatorname{add}(V) \subseteq \operatorname{Refl}_{\zeta}$;

(c) $\mathrm{F}(\operatorname{add}(U))=\operatorname{add}(V)$ and $\mathrm{G}(\operatorname{add}(V))=\operatorname{add}(U)$.

We recall that, a complex $(\mathcal{C}, d)$ in $\mathcal{A}$ is a sequence of objects and morphisms in $\mathcal{A}$

$$
\mathcal{C}: \ldots \stackrel{d_{n-1}}{\longrightarrow} C_{n-1} \stackrel{d_{n}}{\longrightarrow} C_{n} \stackrel{d_{n+1}}{\longrightarrow} C_{n+1} \stackrel{d_{n+2}}{\longrightarrow} \ldots
$$

such that $d_{n+1} d_{n}=0$, for all $n \in \mathbb{Z}$. The morphisms $d_{n}$ are called differentiations. We will shorten the notation $(\mathcal{C}, d)$ to $\mathcal{C}$. We mention that the equation $d_{n+1} d_{n}=0$ is equivalent to $\operatorname{Im}\left(d_{n}\right) \subseteq \operatorname{Ker}\left(d_{n+1}\right)$. Moreover, the complex $\mathcal{C}$ is said to be bounded below (respectively, bounded above), if $C_{n}=0$, for all $n<0$ (respectively, for all $n>0$ ). If $\mathcal{C}$ and $\mathcal{C}^{\prime}$ are two complexes in $\mathcal{A}$, a sequence of morphisms $f=\left(\ldots, f_{n-1}, f_{n}, f_{n+1}, \ldots\right)$, where $f_{n} \in \operatorname{Hom}_{\mathcal{A}}\left(C_{n}, C_{n}^{\prime}\right)$, is called chain map between complexes $\mathcal{C}$ and $\mathcal{C}^{\prime}$ if the following diagram is commutative

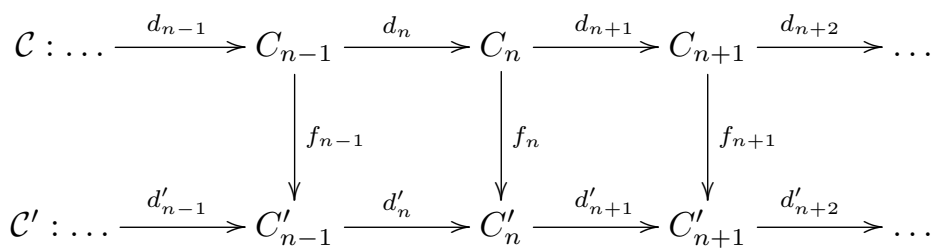

i.e. $f_{n} d_{n}=d_{n}^{\prime} f_{n-1}$, for all integers $n \in \mathbb{Z}$. By $\operatorname{Comp}_{\mathcal{A}}$ will be denoted the category of all complexes in $\mathcal{A}$, defined as follows: the class of objects consist in the class of all 
complexes in $\mathcal{A}$ and the set of morphisms between two complexes $\mathcal{C}$ and $\mathcal{C}^{\prime}$ consist in the set of all chain maps between $\mathcal{C}$ and $\mathcal{C}^{\prime}$.

If $f=\left(\ldots, f_{n-1}, f_{n}, f_{n+1}, \ldots\right): \mathcal{C} \rightarrow \mathcal{C}^{\prime}$ is a chain map between complexes $\mathcal{C}$ and $\mathcal{C}^{\prime}$, then we say that $f$ is null homotopic (or, $f$ is homotopic to zero) if there are, for all integers $n \in \mathbb{Z}$, the morphisms $s_{n}: C_{n} \rightarrow C_{n-1}^{\prime}$ in $\mathcal{A}$ such that $f_{n}=s_{n+1} d_{n+1}+d_{n}^{\prime} s_{n}$, for all integers $n \in \mathbb{Z}$. The sequence $s=\left(\ldots, s_{n-1}, s_{n}, s_{n+1}, \ldots\right)$ is called a homotopy of $f$ (or, a homotopy between $f$ and 0 ). The morphisms are illustrated in the following diagram

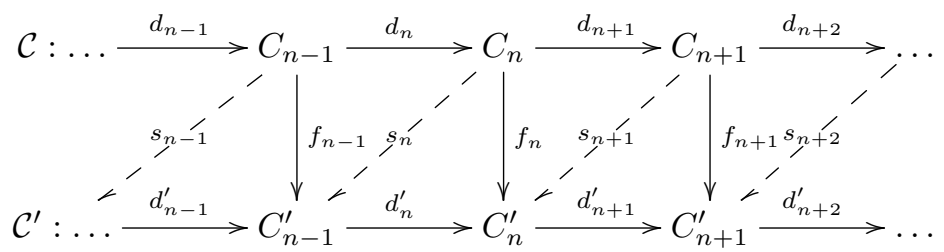

The condition for $s$ to be a homotopy of $f$ says that each vertical morphism is the sum of the sides of the parallelogram containing it. If $f=\left(\ldots, f_{n-1}, f_{n}, f_{n+1}, \ldots\right): \mathcal{C} \rightarrow \mathcal{C}^{\prime}$ and $g=\left(\ldots, g_{n-1}, g_{n}, g_{n+1}, \ldots\right): \mathcal{C} \rightarrow \mathcal{C}^{\prime}$ are two chain maps, then we say that $f$ and $g$ are homotopic (or, $f$ is homotopic to $g$ ), written $f \simeq g$, if

$$
f-g=\left(\ldots, f_{n-1}-g_{n-1}, f_{n}-g_{n}, f_{n+1}-g_{n+1}, \ldots\right): \mathcal{C} \rightarrow \mathcal{C}^{\prime}
$$

is a null homotopic chain map. A homotopy between $f-g$ and 0 is also called a homotopy between $f$ and $g$. The homotopic relation " $\simeq$ is an equivalence relation on the set of chain maps $f: \mathcal{C} \rightarrow \mathcal{C}^{\prime}$. We denote by $[f]$ the homotopy (equivalence) class of $f$.

For a complex $\mathcal{C} \in \operatorname{Comp}_{\mathcal{A}}$ and for some integer $n \in \mathbb{Z}$, we denote by $H_{n}(\mathcal{C})$ the n-th homology of $\mathcal{C}$, i.e. $H_{n}(\mathcal{C})=\operatorname{Ker}\left(d_{n+1}\right) / \operatorname{Im}\left(d_{n}\right)$.

Definition 2.4. Let $\mathcal{U}$ be a class of objects in $\mathcal{A}$. A bounded below complex $\mathcal{C}$ in $\operatorname{Comp}_{\mathcal{A}}$

$$
\mathcal{C}: C_{0} \stackrel{\sigma_{1}}{\longrightarrow} C_{1} \stackrel{\sigma_{2}}{\longrightarrow} C_{2} \stackrel{\sigma_{3}}{\longrightarrow} \ldots
$$

is called $(\mathrm{F}, \mathcal{U})$-coplex if the following conditions are satisfied:

(1) $C_{k} \in \mathcal{U}$, for all $k \geq 0$;

(2) The induced complex

$$
\mathrm{F}(\mathcal{C}): \ldots \stackrel{\mathrm{F}\left(\sigma_{3}\right)}{\longrightarrow} \mathrm{F}\left(C_{2}\right) \stackrel{\mathrm{F}\left(\sigma_{2}\right)}{\longrightarrow} \mathrm{F}\left(C_{1}\right) \stackrel{\mathrm{F}\left(\sigma_{1}\right)}{\longrightarrow} \mathrm{F}\left(C_{0}\right)
$$

is an exact sequence in $\mathcal{B}$.

Now, for a class $\mathcal{U}$ of objects in $\mathcal{A}$, we define the category of all $(\mathrm{F}, \mathcal{U})$-coplexes, denoted by $(\mathrm{F}, \mathcal{U})$-coplex, as follows:

(A) the class of objects consists in the class of all $(\mathrm{F}, \mathcal{U})$-coplexes $\mathcal{C}$;

(B) the set of morphisms between two $(\mathrm{F}, \mathcal{U})$-coplexes $\mathcal{C}$ and $\mathcal{C}^{\prime}$, consists in the set of all homotopy classes of chain maps $f: \mathcal{C} \rightarrow \mathcal{C}^{\prime}$. 
For the rest of the paper, we set a $\delta$-reflexive object $U$ in $\mathcal{A}$ such that $V=\mathrm{F}(U)$ is a projective object in $\mathcal{B}$. Moreover, we suppose that all considered subcategories of $\mathcal{A}$ and $\mathcal{B}$ are isomorphically closed.

Let $Y$ and $B$ be two objects in $\mathcal{B}$ and let $n$ be a positive integer. A projective resolution $\cdots \rightarrow P_{1} \rightarrow P_{0} \rightarrow Y \rightarrow 0$ of $Y$ is called finitely- $B$-generated if $P_{i} \in \operatorname{add}(B)$ for all $i \geq 0$. We will denote by gen $\bullet(B)$ the class of all objects $X \in \mathcal{B}$ such that there exists a finitely- $B$-generated projective resolution of $X$. A projective resolution $\cdots \rightarrow P_{n+1} \rightarrow P_{n} \rightarrow P_{n-1} \rightarrow \cdots \rightarrow P_{1} \rightarrow P_{0} \rightarrow Y \rightarrow 0$ of $Y$ is called $n$-finitely- $B$ generated if $P_{i} \in \operatorname{add}(B)$ for all $i=\overline{0, n}$. We will denote by $n$-gen $\bullet(B)$ the class of all objects $X \in \mathcal{B}$ for which there exists an $n$-finitely- $B$-generated projective resolution of $X$.

Lemma 2.5. Let $\mathcal{C}: C_{0} \stackrel{\sigma_{1}}{\longrightarrow} C_{1} \stackrel{\sigma_{2}}{\longrightarrow} C_{2} \stackrel{\sigma_{3}}{\longrightarrow} \ldots$ be a complex in $\mathrm{Comp}_{\mathcal{A}}$, with $C_{k} \in$ $\operatorname{add}(U)$, for all $k \geq 0$. Then $\mathcal{C}$ is an $(\mathrm{F}$, add $(U))$-coplex if and only if $\mathrm{F}(\mathcal{C})$ is a finitely-V-generated projective resolution of $H_{0}(\mathrm{~F}(\mathcal{C}))$.

Proof. Suppose that $\mathcal{C}$ is an $(\mathrm{F}, \operatorname{add}(U))$-coplex. Then, by definition, the induced sequence

$$
\mathrm{F}(\mathcal{C}): \ldots \stackrel{\mathrm{F}\left(\sigma_{3}\right)}{\longrightarrow} \mathrm{F}\left(C_{2}\right) \stackrel{\mathrm{F}\left(\sigma_{2}\right)}{\longrightarrow} \mathrm{F}\left(C_{1}\right) \stackrel{\mathrm{F}\left(\sigma_{1}\right)}{\longrightarrow} \mathrm{F}\left(C_{0}\right) \stackrel{\varepsilon_{0}}{\longrightarrow} \operatorname{Coker}\left(\mathrm{F}\left(\sigma_{1}\right)\right) \rightarrow 0
$$

is an exact sequence in $\mathcal{B}$. Since all $C_{k} \in \operatorname{add}(U)$, we have, by Lemma 2.3, that all $\mathrm{F}\left(C_{k}\right) \in \operatorname{add}(V)$. We also have that all $\mathrm{F}\left(C_{k}\right)$ are projective in $\mathcal{B}$, because $V$ is projective in $\mathcal{B}$. Therefore $\mathrm{F}(\mathcal{C})$ is a finitely- $V$-generated projective resolution of $\operatorname{Coker}\left(\mathrm{F}\left(\sigma_{1}\right)\right)$.

Conversely, if the induced sequence $\mathrm{F}(\mathcal{C})$ is a finitely- $V$-generated projective resolution of $\operatorname{Coker}\left(\mathrm{F}\left(\sigma_{1}\right)\right)$, then $\mathrm{F}(\mathcal{C})$ is an exact sequence in $\mathcal{B}$. From hypothesis, $C_{k} \in \operatorname{add}(U)$, for all $k \geq 0$. It follows that $\mathcal{C}$ is an $(\mathrm{F}, \operatorname{add}(U))$-coplex.

It is well known that, if $f, g: \mathcal{C} \rightarrow \mathcal{C}^{\prime}$ are two homotopic chain maps between complexes $\mathcal{C}$ and $\mathcal{C}^{\prime}$, then $H_{0}(\mathrm{~F}(f))=H_{0}(\mathrm{~F}(g))$. Therefore, the functor $\mathrm{F}^{U}$ from the following definition is well-defined.

Definition 2.6. The contravariant functor $\mathrm{F}^{U}:(\mathrm{F}, \operatorname{add}(U))$-coplex $\rightarrow \operatorname{gen} \bullet(V)$ is defined as follows:

(A) On objects, we set $\mathrm{F}^{U}(\mathcal{C})=H_{0}(\mathrm{~F}(\mathcal{C}))$, for each $\mathcal{C} \in(\mathrm{F}$, add $(U))$-coplex.

(B) On morphisms, we take $\mathrm{F}^{U}([f])=H_{0}(\mathrm{~F}(f))$, for each morphism $[f]: \mathcal{C} \rightarrow \mathcal{C}^{\prime}$ of $(\mathrm{F}, \operatorname{add}(U))$-coplexes.

Definition 2.7. The contravariant functor $\mathrm{G}^{U}: \operatorname{gen}^{\bullet}(V) \rightarrow(\mathrm{F}$, add $(U))$-coplex is defined as follows:

(A) On objects. Let $Y \in$ gen $^{\bullet}(V)$. Then $Y$ has a finitely- $V$-generated projective resolution

$$
\mathcal{P}(Y): \ldots \stackrel{\partial_{3}}{\longrightarrow} P_{2} \stackrel{\partial_{2}}{\longrightarrow} P_{1} \stackrel{\partial_{1}}{\longrightarrow} P_{0} \stackrel{\partial_{0}}{\longrightarrow} Y \rightarrow 0 \text {. }
$$

We mention that the chosen projective resolution $\mathcal{P}(Y)$ is unique up to a homotopy. Applying the functor $\mathrm{G}$ to the projective resolution $\mathcal{P}(Y)$, we obtain the following complex in $\mathcal{A}$

$$
\mathrm{G}(\mathcal{P}(Y)): \mathrm{G}\left(P_{0}\right) \stackrel{\mathrm{G}\left(\partial_{1}\right)}{\longrightarrow} \mathrm{G}\left(P_{1}\right) \stackrel{\mathrm{G}\left(\partial_{2}\right)}{\longrightarrow} \mathrm{G}\left(P_{2}\right) \stackrel{\mathrm{G}\left(\partial_{3}\right)}{\longrightarrow} \ldots
$$


Since $\mathcal{P}(Y)$ is finitely- $V$-generated, we have $P_{k} \in \operatorname{add}(V)$, for all $k \geq 0$, and, since $\zeta: 1_{\operatorname{add}(V)} \rightarrow \mathrm{FG}$ is a natural isomorphism, the following diagram is commutative with the vertical maps isomorphisms

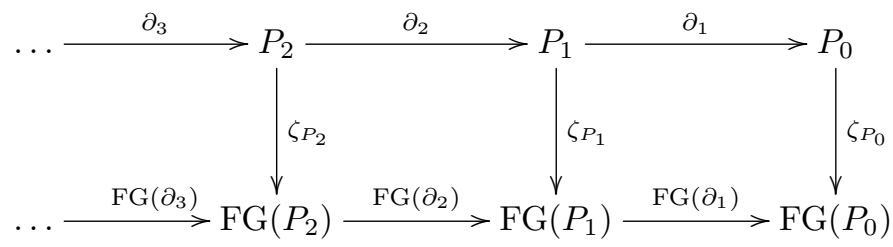

Since the top row is an exact sequence, it follows that the bottom row is an exact sequence. By Lemma 2.3, $\mathrm{G}\left(P_{k}\right) \in \operatorname{add}(U)$, for all $k \geq 0$. Thus $\mathrm{G}(\mathcal{P}(Y))$ is a complex in $\mathcal{A}$ with all $\mathrm{G}\left(P_{k}\right) \in \operatorname{add}(U)$ and the induced sequence $\operatorname{FG}(\mathcal{P}(Y))$ is an exact sequence. Therefore $\mathrm{G}(\mathcal{P}(Y))$ is an $(\mathrm{F}$, add $(U))$-coplex. We set

$$
\mathrm{G}^{U}(Y)=\mathrm{G}(\mathcal{P}(Y)) \text {. }
$$

(B) On morphisms. Let $\phi \in \operatorname{Hom}_{\text {gen }}(V)\left(Y, Y^{\prime}\right)$. Then $\phi$ lifts to a chain map

$$
f=\left(\ldots, f_{2}, f_{1}, f_{0}\right): \mathcal{P}(Y) \rightarrow \mathcal{P}\left(Y^{\prime}\right)
$$

where $\mathcal{P}(Y)$ and $\mathcal{P}\left(Y^{\prime}\right)$ are finitely- $V$-generated projective resolutions associated to $Y$ and $Y^{\prime}$, respectively.

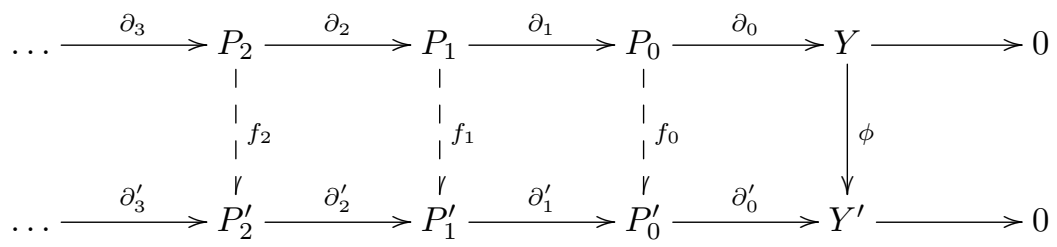

Applying the functor $\mathrm{G}$, we get a chain map in $\mathcal{A}$,

$$
\mathrm{G}(f)=\left(\mathrm{G}\left(f_{0}\right), \mathrm{G}\left(f_{1}\right), \mathrm{G}\left(f_{2}\right), \ldots\right): \mathrm{G}\left(\mathcal{P}\left(Y^{\prime}\right)\right) \rightarrow \mathrm{G}(\mathcal{P}(Y))
$$

illustrated in the following diagram

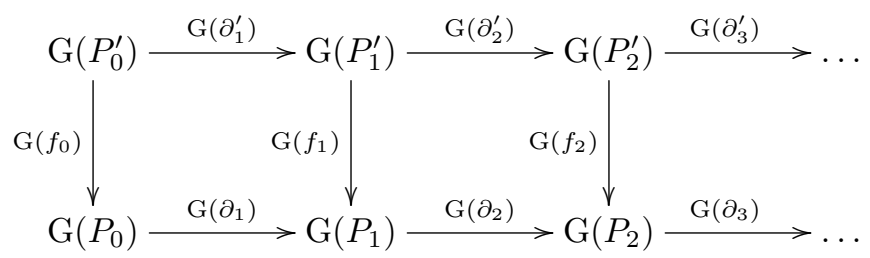

Since $\mathrm{G}(\mathcal{P}(Y))$ and $\mathrm{G}\left(\mathcal{P}\left(Y^{\prime}\right)\right)$ are $(\mathrm{F}$, add $(U))$-coplexes, it follows that the homotopy class $[\mathrm{G}(f)]$ is a morphism in the category $(\mathrm{F}$, add $(U))$-coplex. We set

$$
\mathrm{G}^{U}(\phi)=[\mathrm{G}(f)]
$$




\section{Main result}

The main result of the paper is the following theorem.

Theorem 3.1. The functors $\mathrm{F}^{U}$ and $\mathrm{G}^{U}$ induce the following duality

$$
\mathrm{F}^{U}:(\mathrm{F}, \operatorname{add}(U)) \text {-coplex } \rightleftarrows \operatorname{gen} \bullet(V): \mathrm{G}^{U}
$$

Proof. First, we show that the composition $\mathrm{F}^{U} \circ \mathrm{G}^{U}$ is natural isomorphic to the identity functor $1_{\text {gen } \bullet}(V)$.

Let $Y \in \operatorname{gen}^{\bullet}(V)$. Then $Y$ has a finitely- $V$-generated projective resolution

$$
\mathcal{P}(Y): \ldots \stackrel{\partial_{3}}{\longrightarrow} P_{2} \stackrel{\partial_{2}}{\longrightarrow} P_{1} \stackrel{\partial_{1}}{\longrightarrow} P_{0} \stackrel{\partial_{0}}{\longrightarrow} Y \rightarrow 0 .
$$

Applying the functor $\mathrm{G}$, we obtain the following $(\mathrm{F}$, $\operatorname{add}(U))$-coplex

$$
\mathrm{G}(\mathcal{P}(Y)): \mathrm{G}\left(P_{0}\right) \stackrel{\mathrm{G}\left(\partial_{1}\right)}{\longrightarrow} \mathrm{G}\left(P_{1}\right) \stackrel{\mathrm{G}\left(\partial_{2}\right)}{\longrightarrow} \mathrm{G}\left(P_{2}\right) \stackrel{\mathrm{G}\left(\partial_{3}\right)}{\longrightarrow} \ldots
$$

and then $\mathrm{G}^{U}(Y)=\mathrm{G}(\mathcal{P}(Y))$. Applying the functor $\mathrm{F}$, we have the exact sequence $\mathrm{FG}(\mathcal{P}(Y)): \ldots \stackrel{\mathrm{FG}\left(\partial_{3}\right)}{\longrightarrow} \mathrm{FG}\left(P_{2}\right) \stackrel{\mathrm{FG}\left(\partial_{2}\right)}{\longrightarrow} \mathrm{FG}\left(P_{1}\right) \stackrel{\mathrm{FG}\left(\partial_{1}\right)}{\longrightarrow} \mathrm{FG}\left(P_{0}\right) \stackrel{\varepsilon_{0}}{\longrightarrow} \operatorname{Coker}\left(\mathrm{FG}\left(\partial_{1}\right)\right) \rightarrow 0$ and then $\mathrm{F}^{U}(\mathrm{G}(\mathcal{P}(Y)))=\operatorname{Coker}\left(\mathrm{FG}\left(\partial_{1}\right)\right)$. Thus $\left(\mathrm{F}^{U} \circ \mathrm{G}^{U}\right)(Y)=\operatorname{Coker}\left(\mathrm{FG}\left(\partial_{1}\right)\right)$.

Since all $P_{k} \in \operatorname{add}(V)$ and since $\zeta: 1_{\operatorname{add}(V)} \rightarrow \mathrm{FG}$ is a natural isomorphism, the following diagram is commutative with the vertical maps isomorphisms.

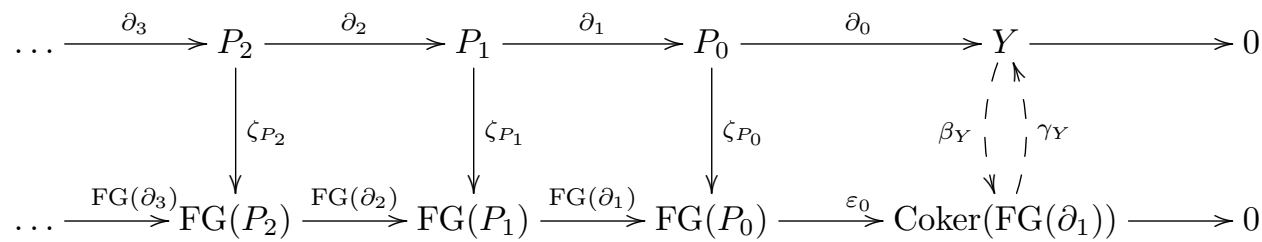

Since $\left(\varepsilon_{0} \circ \zeta_{P_{0}}\right) \circ \partial_{1}=0$ and $Y$ is the cokernel of $\partial_{1}$, there is a unique morphism $\beta_{Y}: Y \rightarrow \operatorname{Coker}\left(\mathrm{FG}\left(\partial_{1}\right)\right)$ such that $\varepsilon_{0} \circ \zeta_{P_{0}}=\beta_{Y} \circ \partial_{0}$. Also, since $\left(\partial_{0} \circ \zeta_{P_{0}}^{-1}\right) \circ \mathrm{FG}\left(\partial_{1}\right)=$ 0 , there is a unique morphism $\gamma_{Y}: \operatorname{Coker}\left(\mathrm{FG}\left(\partial_{1}\right)\right) \rightarrow Y$ such that $\partial_{0} \circ \zeta_{P_{0}}^{-1}=\gamma_{Y} \circ \varepsilon_{0}$. It it easy to see that $\beta_{Y} \circ \gamma_{Y}=1_{\mathrm{Coker}\left(\mathrm{FG}\left(\partial_{1}\right)\right)}$ and $\gamma_{Y} \circ \beta_{Y}=1_{Y}$. Thus $\beta_{Y}: Y \rightarrow$ $\left(\mathrm{F}^{U} \circ \mathrm{G}^{U}\right)(Y)$ is an isomorphism.

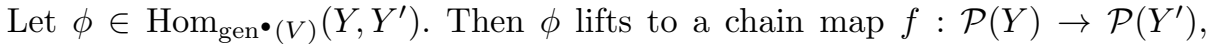
where $\mathcal{P}(Y)$ and $\mathcal{P}\left(Y^{\prime}\right)$ are the finitely- $V$-generated projective resolutions of $Y$ and $Y^{\prime}$, respectively, as we see in the following diagram:

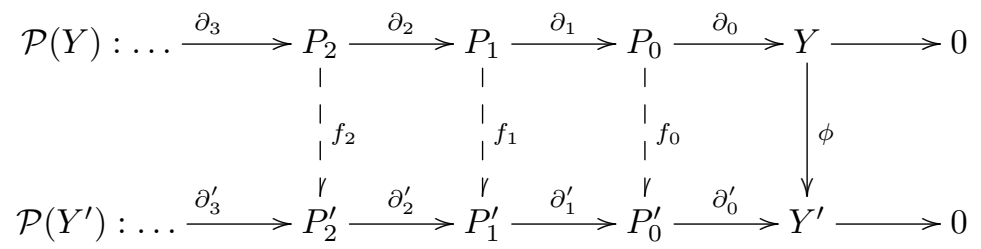

By definition, we have $\mathrm{G}^{U}(\phi)=[\mathrm{G}(f)]: \mathrm{G}^{U}\left(Y^{\prime}\right) \rightarrow \mathrm{G}^{U}(Y)$. 


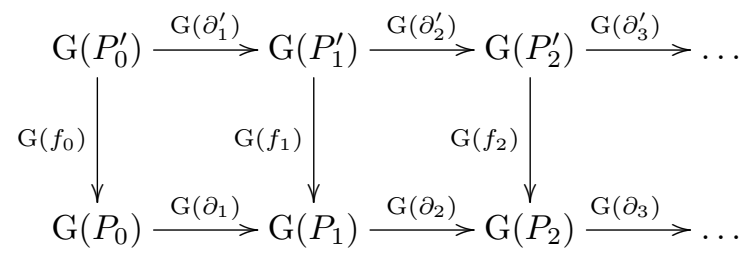

Since $\varepsilon_{0}^{\prime} \circ \mathrm{FG}\left(f_{0}\right) \circ \mathrm{FG}\left(\partial_{1}\right)=0$, there is a unique morphism $\alpha$ : $\operatorname{Coker}\left(\mathrm{FG}\left(\partial_{1}\right)\right) \rightarrow$ $\operatorname{Coker}\left(\mathrm{FG}\left(\partial_{1}^{\prime}\right)\right)$ such that $\varepsilon_{0}^{\prime} \circ \mathrm{FG}\left(f_{0}\right)=\alpha \circ \varepsilon_{0}$. Then $\mathrm{F}^{U}([\mathrm{G}(f)])=\alpha$, and thus $\left(\mathrm{F}^{U} \circ \mathrm{G}^{U}\right)(\phi)=\alpha$.

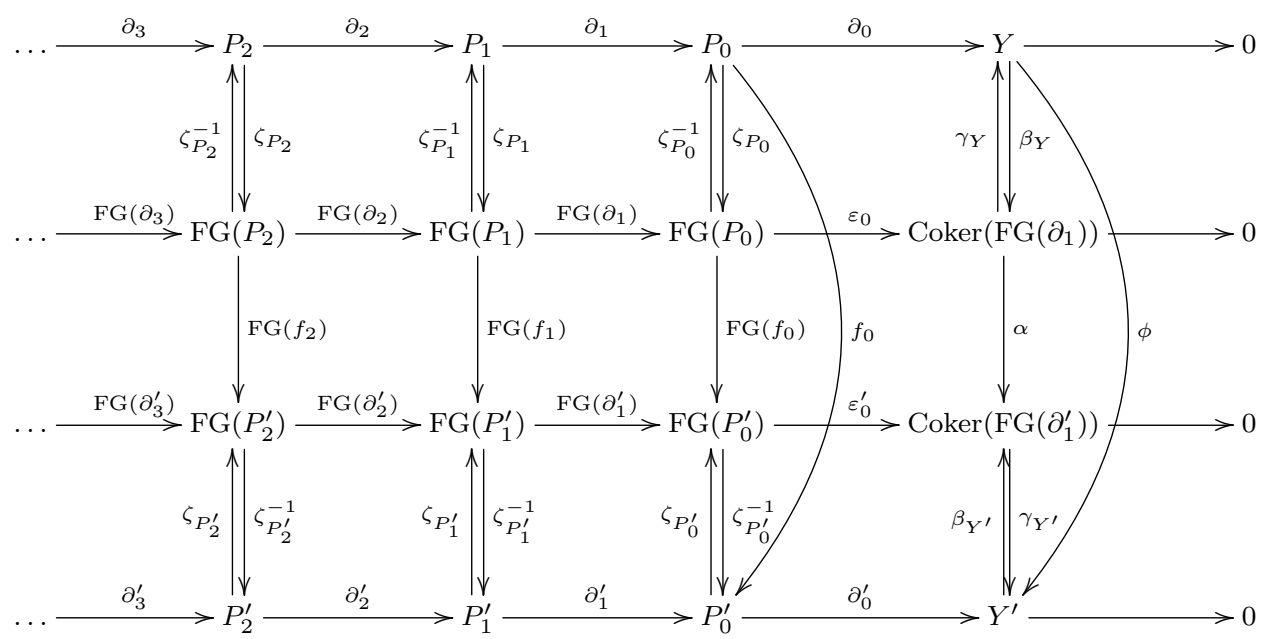

From the fact that $\zeta: 1_{\mathcal{B}} \rightarrow \mathrm{FG}$ is a natural transformation, we have $\mathrm{FG}\left(f_{0}\right) \circ$ $\zeta_{P_{0}}=\zeta_{P_{0}^{\prime}} \circ f_{0}$. It follows that we have the following equalities

$$
\begin{gathered}
\alpha \circ \beta_{Y} \circ \partial_{0}=\alpha \circ \varepsilon_{0} \circ \zeta_{P_{0}}= \\
\varepsilon_{0}^{\prime} \circ \mathrm{FG}\left(f_{0}\right) \circ \zeta_{P_{0}}=\varepsilon_{0}^{\prime} \circ \zeta_{P_{0}^{\prime}} \circ f_{0}= \\
\beta_{Y^{\prime}} \circ \partial_{0}^{\prime} \circ f_{0}=\beta_{Y^{\prime}} \circ \phi \circ \partial_{0} .
\end{gathered}
$$

Hence $\alpha \circ \beta_{Y}=\beta_{Y^{\prime}} \circ \phi$, because $\partial_{0}$ is an epimorphism. Therefore we have the equality $\left(\mathrm{F}^{U} \circ \mathrm{G}^{U}\right)(\phi) \circ \beta_{Y}=\beta_{Y^{\prime}} \circ \phi$, i.e. the following diagram is commutative

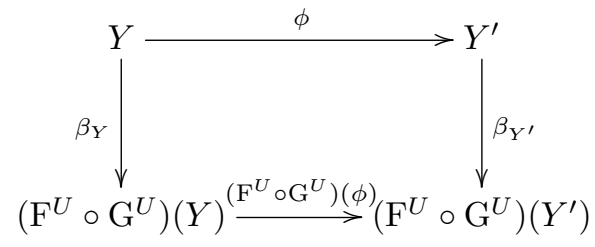

Second, we show that the composition $\mathrm{G}^{U} \circ \mathrm{F}^{U}$ is natural isomorphic with the identity functor $1_{(\mathrm{F}, \operatorname{add}(U)) \text {-coplex }}$. 
Let $\mathcal{C} \in(\mathrm{F}, \operatorname{add}(U))$-coplex. Then

$$
\mathcal{C}: C_{0} \stackrel{\sigma_{1}}{\longrightarrow} C_{1} \stackrel{\sigma_{2}}{\longrightarrow} C_{2} \stackrel{\sigma_{3}}{\longrightarrow} \ldots
$$

is a complex in $\mathcal{A}$, with $C_{k} \in \operatorname{add}(U)$, for all $k \geq 0$, and the induced sequence

$$
\mathrm{F}(\mathcal{C}): \ldots \stackrel{\mathrm{F}\left(\sigma_{3}\right)}{\longrightarrow} \mathrm{F}\left(C_{2}\right) \stackrel{\mathrm{F}\left(\sigma_{2}\right)}{\longrightarrow} \mathrm{F}\left(C_{1}\right) \stackrel{\mathrm{F}\left(\sigma_{1}\right)}{\longrightarrow} \mathrm{F}\left(C_{0}\right) \stackrel{\varepsilon_{0}}{\longrightarrow} \operatorname{Coker}\left(\mathrm{F}\left(\sigma_{1}\right)\right) \rightarrow 0
$$

is a finitely- $V$-generated projective resolution of $\operatorname{Coker}\left(\mathrm{F}\left(\sigma_{1}\right)\right)$. By definition $\mathrm{F}^{U}(\mathcal{C})=$ $\operatorname{Coker}\left(\mathrm{F}\left(\sigma_{1}\right)\right)$. Moreover, $\mathrm{G}^{U}\left(\operatorname{Coker}\left(\mathrm{F}\left(\sigma_{1}\right)\right)\right)=\mathrm{GF}(\mathcal{C})$, hence $\left(\mathrm{G}^{U} \circ \mathrm{F}^{U}\right)(\mathcal{C})=\mathrm{GF}(\mathcal{C})$.

Since $\delta: 1_{\mathcal{A}} \rightarrow \mathrm{GF}$ is a natural transformation, we have that

$$
\delta_{\mathcal{C}}=\left(\delta_{C_{0}}, \delta_{C_{1}}, \delta_{C_{2}}, \ldots\right)
$$

is a chain map between $\left(\mathrm{F}\right.$, add $(U)$ )-coplexes $\mathcal{C}$ and $\operatorname{GF}(\mathcal{C})$, hence we have $\left[\delta_{\mathcal{C}}\right] \in$ $\operatorname{Hom}_{(\mathrm{F}, \operatorname{add}(U)) \text {-coplex }}(\mathcal{C}, \operatorname{GF}(\mathcal{C}))$. On the other hand, since $C_{k} \in \operatorname{add}(U)$, the morphisms $\delta_{C_{k}}: C_{k} \rightarrow \mathrm{GF}\left(C_{k}\right)$ are isomorphisms, hence

$$
\delta_{\mathcal{C}}^{-1}=\left(\delta_{C_{0}}^{-1}, \delta_{C_{1}}^{-1}, \delta_{C_{2}}^{-1}, \ldots\right)
$$

is a chain map between $(\mathrm{F}, \operatorname{add}(U))$-coplexes $\operatorname{GF}(\mathcal{C})$ and $\mathcal{C}$ and thus we have $\left[\delta_{\mathcal{C}}^{-1}\right] \in$ $\operatorname{Hom}_{(\mathrm{F}, \operatorname{add}(U)) \text {-coplex }}(\mathrm{GF}(\mathcal{C}), \mathcal{C})$.

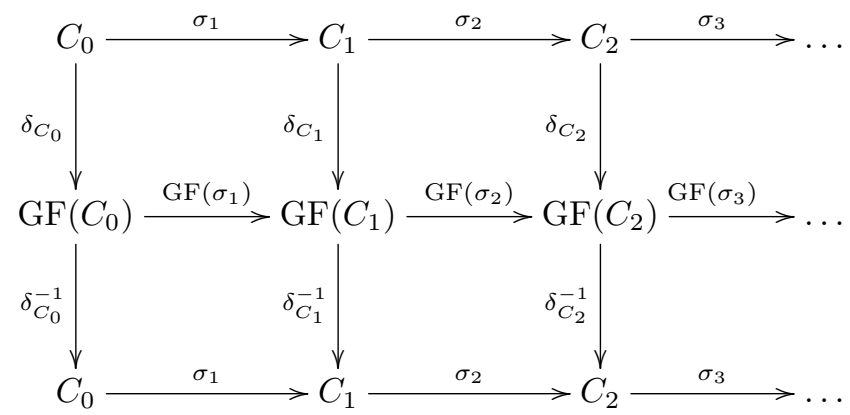

Since $\delta_{C_{k}}^{-1} \circ \delta_{C_{k}}=1_{C_{k}}$ and $\delta_{C_{k}} \circ \delta_{C_{k}}^{-1}=1_{\mathrm{GF}\left(C_{k}\right)}$ in $\mathcal{A}$, for all $k \geq 0$, we have $\left[\delta_{\mathcal{C}}^{-1}\right] \circ\left[\delta_{\mathcal{C}}\right]=$ $\left[1_{\mathcal{C}}\right]$ and $\left[\delta_{\mathcal{C}}\right] \circ\left[\delta_{\mathcal{C}}^{-1}\right]=\left[1_{\mathrm{GF}(\mathcal{C})}\right]$ in $(\mathrm{F}, \operatorname{add}(U))$-coplex, hence $\left[\delta_{\mathcal{C}}\right]: \mathcal{C} \rightarrow\left(\mathrm{G}^{U} \circ \mathrm{F}^{U}\right)(\mathcal{C})$ is an isomorphism in $(\mathrm{F}, \operatorname{add}(U))$-coplex.

Let $[f] \in \operatorname{Hom}_{(\mathrm{F}, \operatorname{add}(U)) \text {-coplex }}\left(\mathcal{C}, \mathcal{C}^{\prime}\right)$. Then

$$
f=\left(f_{0}, f_{1}, f_{2}, \ldots\right): \mathcal{C} \rightarrow \mathcal{C}^{\prime}
$$

is a chain map between $(\mathrm{F}, \operatorname{add}(U))$-coplexes $\mathcal{C}$ and $\mathcal{C}^{\prime}$, as illustrated below:

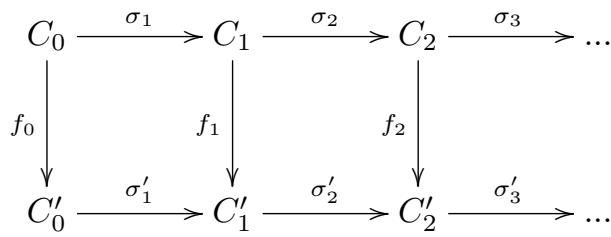

It follows that

$$
\mathrm{F}(f)=\left(\ldots, \mathrm{F}\left(f_{2}\right), \mathrm{F}\left(f_{1}\right), \mathrm{F}\left(f_{0}\right)\right): \mathrm{F}\left(\mathcal{C}^{\prime}\right) \rightarrow \mathrm{F}(\mathcal{C})
$$


is a chain map between exact sequences $\mathrm{F}\left(\mathcal{C}^{\prime}\right)$ and $\mathrm{F}(\mathcal{C})$

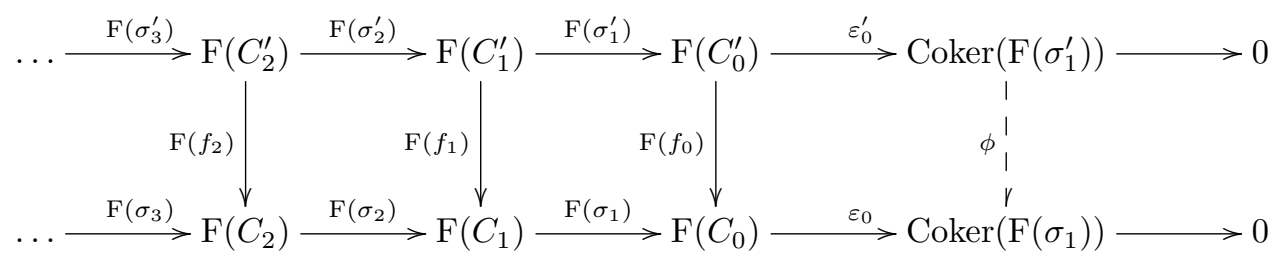

Since $\left(\varepsilon_{0} \circ \mathrm{F}\left(f_{0}\right)\right) \circ \mathrm{F}\left(\sigma_{1}^{\prime}\right)=0$, there is a unique morphism $\phi: \operatorname{Coker}\left(\mathrm{F}\left(\sigma_{1}^{\prime}\right)\right) \rightarrow$ $\operatorname{Coker}\left(\mathrm{F}\left(\sigma_{1}\right)\right)$ in $\mathcal{B}$ such that $\varepsilon_{0} \circ \mathrm{F}\left(f_{0}\right)=\phi \circ \varepsilon_{0}^{\prime}$ and then, by definition, $\mathrm{F}^{U}([f])=\phi$. Moreover, by definition of $\mathrm{G}^{U}$, we have $\mathrm{G}^{U}(\phi)=[\mathrm{GF}(f)]$. Thus $\left(\mathrm{G}^{U} \circ \mathrm{F}^{U}\right)([f])=$ $[\mathrm{GF}(f)]$.

Since $\delta: 1_{\mathcal{A}} \rightarrow \mathrm{GF}$ is a natural transformation, we have $\mathrm{GF}\left(f_{k}\right) \circ \delta_{C_{k}}=\delta_{C_{k}^{\prime}} \circ f_{k}$, for all $k \geq 0$, hence $\left[\mathrm{GF}(f) \circ \delta_{\mathcal{C}}\right]=\left[\delta_{\mathcal{C}^{\prime}} \circ f\right]$. Thus $[\mathrm{GF}(f)] \circ\left[\delta_{\mathcal{C}}\right]=\left[\delta_{\mathcal{C}^{\prime}}\right] \circ[f]$ and therefore $\left(\mathrm{G}^{U} \circ \mathrm{F}^{U}\right)([f]) \circ\left[\delta_{\mathcal{C}}\right]=\left[\delta_{\mathcal{C}^{\prime}}\right] \circ[f]$. So, the following diagram is commutative

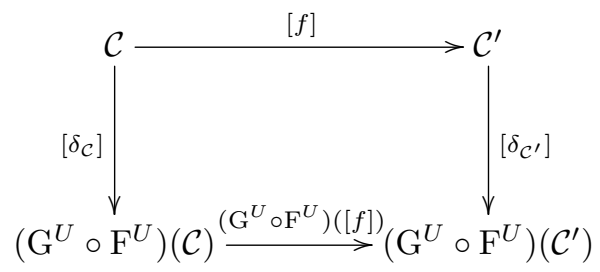

Acknowledgement. I would like to mention that this paper is a part of my $\mathrm{PhD}$ thesis, supervised by Professor Andrei Marcus. Furthermore, I present my thanks to the referees for their comments and suggestions that improved this article.

\section{References}

[1] Al-Nofayee, S., r-Costar pair of contravariant functors, International Journal of Mathematics and Mathematical Sciences, 2012, Article ID 481909, 8 pages.

[2] Al-Nofayee, S., Co- $\star^{n}$-tuple of contravariant functors, British Journal of Mathematics and Computer Science, 4(2014), no. 12, 1701-1709.

[3] Al-Nofayee, S., $\star^{s}$-tuple and $\star^{n}$-tuple of covariant functors, British Journal of Mathematics and Computer Science, 7(2015), no. 6, 439-449.

[4] Breaz, S., Finitistic n-self-cotilting modules, Communications in Algebra, 37(2009), no. 9, 3152-3170.

[5] Breaz, S., Modoi, C., Pop, F., Natural Equivalences and Dualities, Proceedings of the International Conference on Modules and Representation Theory (7-12 July 2008, ClujNapoca, Romania), Cluj University Press, 2009, 25-40.

[6] Breaz, S., Pop, F., Dualities Induced by Right Adjoint Contravariant Functors, Stud. Univ. Babeş-Bolyai Math., 55(2010), no. 1, 75-83.

[7] Castaño-Iglesias, F., On a natural duality between Grothendieck categories, Communications in Algebra, 36(2008), no. 6, 2079-2091. 
[8] Colby, R.R., Fuller, K.R., Costar modules, Journal of Algebra, 242(2001), no. 1, 146-159.

[9] Colby, R.R., Fuller, K.R., Equivalence and Duality for Module Categories. With Tilting and Cotilting for Rings, Cambridge University Press, Cambridge, 2004.

[10] Faticoni, T.G., A duality for self-slender modules, Communications in Algebra, 35(2007), no. $12,4175-4182$.

[11] Faticoni, T.G., Modules Over Endomorphism Rings, Cambridge University Press, Cambridge, 2010.

[12] Fuller, K.R., Natural and doubly natural dualities, Communications in Algebra, 34(2006), no. 2, 749-762.

[13] Năstăsescu, C., Van Oystaeyen, F., Methods of Graded Rings, Lectures Notes in Mathematics 1836, Springer-Verlag, 2004.

[14] Pop, F., Natural dualities between abelian categories, Central European Journal of Mathematics, 9(2011), no. 5, 1088-1099.

[15] Pop, F., Closure properties associated to natural equivalences, Indagationes Mathematicae, 24(2013), no. 2, 403-414.

[16] Wisbauer, R., Cotilting objects and dualities, Representations of algebras, Sao Paulo, 1999, 215-233, Lecture Notes in Pure and Appl. Math., 224, Dekker, New York, 2002.

Flaviu Pop

Babeş-Bolyai University

Faculty of Economics and Business Administration

Cluj-Napoca, Romania

e-mail: flaviu.v@gmail.com

flaviu.pop@econ.ubbcluj.ro 